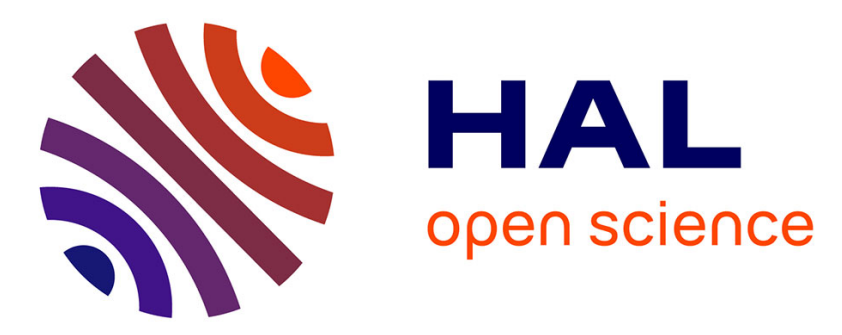

\title{
Examining the effect of geometry changes in industrial fuel injection systems on hydrodynamic structures with biglobal linear stability analysis
}

Thomas Ludwig Kaiser, Kilian Oberleithner, Laurent Selle, Thierry Poinsot

\section{- To cite this version:}

Thomas Ludwig Kaiser, Kilian Oberleithner, Laurent Selle, Thierry Poinsot. Examining the effect of geometry changes in industrial fuel injection systems on hydrodynamic structures with biglobal linear stability analysis. Journal of Engineering for Gas Turbines and Power, 2020, 142 (1), pp.011024. 10.1115/1.4045018 . hal-03292076

\section{HAL Id: hal-03292076 https://hal.science/hal-03292076}

Submitted on 20 Jul 2021

HAL is a multi-disciplinary open access archive for the deposit and dissemination of scientific research documents, whether they are published or not. The documents may come from teaching and research institutions in France or abroad, or from public or private research centers.
L'archive ouverte pluridisciplinaire HAL, est destinée au dépôt et à la diffusion de documents scientifiques de niveau recherche, publiés ou non, émanant des établissements d'enseignement et de recherche français ou étrangers, des laboratoires publics ou privés. 


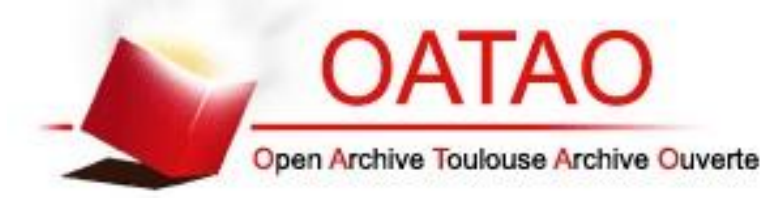

\section{Open Archive Toulouse Archive Ouverte}

OATAO is an open access repository that collects the work of Toulouse researchers and makes it freely available over the web where possible

This is an author's version published in: http://oatao.univ-toulouse.fr/28059

\section{Official URL:}

https://doi.org/10.1115/1.4045018

\section{To cite this version:}

Kaiser, Thomas Ludwig and Oberleithner, Kilian and Selle, Laurent and Poinsot, Thierry Examining the effect of geometry changes in industrial fuel injection systems on hydrodynamic structures with biglobal linear stability analysis. (2020) Journal Of Engineering For Gas Turbines And Power, 142 (1). 011024. ISSN 0742-4795

Any correspondence concerning this service should be sent to the repository administrator: tech-oatao@listes-diff.inp-toulouse.fr 


\section{EXAMINING THE EFFECT OF GEOMETRY CHANGES IN INDUSTRIAL FUEL INJECTION SYSTEMS ON HYDRODYNAMIC STRUCTURES WITH BIGLOBAL LINEAR STABILITY ANALYSIS}

\author{
Thomas Ludwig Kaiser* \\ Laboratory for Flow Instabilities and Dynamics \\ Technische Universität Berlin, \\ Müller-Breslau-Straße 8, \\ 10623 Berlin, Germany \\ Email: t.kaiser@tu-berlin.de
}

\section{Laurent Selle}

Institut de Mécanique des Fluides de Toulouse, IMFT, Université de Toulouse, CNRS - Toulouse, FRANCE

\author{
Kilian Oberleithner \\ Laboratory for Flow Instabilities and Dynamics \\ Technische Universität Berlin, \\ Müller-Breslau-Straße 8, \\ 10623 Berlin, Germany
}

\begin{abstract}
Shape optimization with respect to the suppression or enhancement of dynamical flow structures is an important topic in combustion research and beyond. In this paper, we investigate the flow in an industrial fuel injection system by experimental means, as well as Large Eddy Simulation (LES) and Linear Stability Analysis (LSA) for two configurations of the swirler. In the first configuration, the reference geometry, a Precessing Vortex Core $(P V C)$ occurs. In the second configuration, a center body is mounted in the interior of the injector. It is shown by both experiments and LES that the PVC is suppressed by the presence of the center body, while the mean flow remains nearly unaffected. The method of LSA is applied in order to explain the effect of the geometry change. The work shows that LSA is capable of explaining the occurrence or disappearance of coherent structures evolving on the turbulent flows if the geometry is changed. This is an important step in using LSA in the context of shape optimization of industrial fuel injectors.
\end{abstract}

\footnotetext{
*Address all correspondence to this author.
}

\section{Thierry Poinsot}

Institut de Mécanique des Fluides de Toulouse, IMFT, Université de Toulouse, CNRS - Toulouse, FRANCE CERFACS - Toulouse, FRANCE

\section{NOMENCLATURE}

Latin:

$D \quad$ Characteristic length

$f \quad$ Frequency

$i \quad$ Imaginary number

$K \quad$ Turbulent kinetic energy

$m \quad$ Azimuthal wave number

n Normal vector

$p \quad$ Pressure

Re Reynolds number

$t \quad$ Time

u Velocity

$U \quad$ Characteristic velocity

Greek:

Kronecker delta

Viscosity

$\omega \quad$ Circular frequency 


\section{Superscripts:}

$\begin{array}{ll}\widetilde{\langle\cdot\rangle} & \text { Coherent fluctuation } \\ \frac{\langle\cdot\rangle}{\langle\cdot\rangle^{\prime}} & \text { Fourier transform } \\ \frac{\langle\cdot\rangle}{\langle\cdot\rangle} & \text { Stochastic fluctuation } \\ & \text { Temporal mean }\end{array}$

$\begin{array}{ll} & \text { Subscripts: } \\ \langle\cdot\rangle_{\mathrm{e}} & \text { Eddy } \\ \langle\cdot\rangle_{\mathrm{i}} & \text { Imaginary } \\ \langle\cdot\rangle_{r} & \text { Radial } \\ \langle\cdot\rangle_{\mathrm{r}} & \text { Real } \\ \langle\cdot\rangle_{\mathrm{t}} & \text { Azimuthal } \\ \langle\cdot\rangle_{z} & \text { Axial }\end{array}$

\section{Introduction}

The phenomenon of a Precessing Vortex Core (PVC) in the swirled injection system of a combustion engine has been attracting increasingly the attention of the research community in the recent years. This is due to its beneficial influence on mixing and fuel atomization $[1,2]$. However, coherent structures like the PVC are a source of Combustion Instabilities (CIs) and can couple with an acoustic mode and lead to thermoacoustic instabilities [3]. Therefore, an important goal of contemporary research focusing on the instability is understanding the feedback mechanism and finding related methods of control. A valuable tool in this context is the Linear Stability Analysis (LSA), which is based on the linearization of the governing equations of the fluid flow around its time-average. This method allows the temporal and spatial stability of modal structures to be investigated. Thereby, it not only yields the spatial structure of a mode and its corresponding frequency and growth rate, it also provides the region for optimal forcing. This information is highly valuable in order to find the optimal location for active control of an instability $[4,5]$. Based on such information, Kuhn et al. [6] and Lückoff et al. [7] determined the optimal placements of actuators in the context of open and closed loop control of a PVC.

Furthermore, the region of highest internal feedback of the instability mechanism can be identified by LSA. This region can be seen as the origin of the perturbation and is often referred to as wave maker. Giannetti et al. [5] and Chomaz [8] demonstrated that for the cylinder wake flow, the region of highest internal feedback of the von Kármán vortex street agrees well with the regions for which a localized momentum drag has the strongest stabilizing effect based on the experimental results of Strykowski and Sreenivasan [9]. The localized moment drag in the experiment was introduced by a second, much smaller cylinder, which was traversed through the experimental domain to find the regions where its stabilizing impact is most significant.

A third aspect in the context of control which is related to LSA is shape optimization with respect to the evolution of the

GTP-19-1433 Thomas Ludwig Kaiser coherent structures. Tammisola and Juniper [10] applied the adjoint method based on a BiGlobal LSA in order to identify the effect of localized shift of the wall boundaries into the domain on the growth rate of the PVC. Aimed adaptation of the swirler geometry can therefore be seen as a tool for passive control of the PVC. The question of how well linear stability analysis captures the impact of geometry changes however remains not well addressed.

This paper examines whether LSA correctly describes the influence of a large scale geometry change on the hydrodynamic stability of a PVC in the flow of an industrial, two-staged, counter-rotative fuel injection system. The reference geometry, which from here on is referred to as Configuration A (Conf. A) is illustrated in Fig. 1a. In the second configuration referenced to as Configuration B (Conf. B) a cylindrical rod is mounted coaxially in the interior primary injector (see Fig. 1b). Additionally to the purpose of testing the method of LSA, this geometry change is of large practical importance: These rods are often used as central bluff bodies to support flame anchoring or they are used as a part of the fuel line. As shown by Paschereit et al. [11], injecting additional fuel through a lance, which is - like our rod - mounted on the axis of the injector can significantly reduce thermoacoustic oscillations. On the other hand Paschereit et al. also raise concerns about flash back safety, which are attributed to a center body, and unwanted internal stabilization of the flame on the lance. Therefore, understanding how a central bluff body modifies the mean flow and the flow dynamics is a general question for the combustion community. In this context, we furthermore address the impact of the central rod on the flame shape by reactive Large Eddy Simulation (LES).

The paper is organized as follows: The swirler geometry and the experimental set-up are presented in Section 2. Subsequently, the numerical set-up for both the LESs and the BiGlobal LSA are described in Sections 3.1 and 3.2, respectively. The results of the experimental investigations, of the LES and of the BiGlobal LSA are presented in Section 4, and the results of this study are concluded in Section. 5.

\section{Experimental Set-Up}

The internal geometry of the swirler is illustrated in Fig. 2. The swirler is two staged and counter-rotative. The flow enters both stages through eight tangential vanes. The position of the central rod in Conf. B is shown by the dashed lines in Fig. 2.

Figure 3 shows a sketch of the experimental set-up. A cylindrical upstream plenum of a diameter of $88 \mathrm{~mm}$ and a length of $590 \mathrm{~mm}$ feeds both the primary and secondary swirler vanes. The swirler blows into the free atmosphere. Free stream velocity profiles in the meridional plane are provided by Particle Image Velocimetry (PIV) measurements. Olive oil droplets are used as seeding particles, which are injected in an additional second air line and mixed with the main line in a mixing vessel. A Nd:YAG 
laser illuminates the seeding particles and their scattering light is captured by a window of $20 \mathrm{~mm} \times 32 \mathrm{~mm}$.

\section{Numerical Set-Up}

The numerical strategy involves two independent approaches. Firstly, LES (see Section 3.1) are applied for time integration of the Navier-Stokes equations. The obtained timeaveraged fields serve as input for the second approach, the LSA, the theory of which is addressed in Section 3.2.

\subsection{LES}

The LESs were performed with the AVBP software [12], which is developed at CERFACS and IFPEN. The software is specialized in time-integration of the Navier-Stokes momentum, continuity and energy equations on unstructured meshes. The TTGC scheme [13] is applied, which is of third order accuracy in space and time. The sigma model [14] is used to model the sub-grid turbulence. The computational domain consists of the last $90 \mathrm{~mm}$ of the circular plenum, the swirler and the free atmosphere is modeled by a half-sphere. Figure. 4 shows the numerical mesh exemplary for the coldflow simulations of Conf. A. An iterative mesh h-refinement strategy is applied, which is based on the work of Daviller et al. [15] (see zones of localized high refinement in Fig. 4). In total, the domain is discretized with 10.86 Mio. cells, where the finest cells are of size $45 \mu \mathrm{m}$. The time step is set to a fixed value of $\Delta t=3.3 e-8 \mathrm{~s}$, which yields an approximate maximal Courant-Friedrichs-Lewy (CFL) number of 0.9 . Both at the reflective inlet (reflection coefficient, $K=70000$ ) and at the non-reflective outlet $(K=1000)$ Navier-Stokes Characteristic Boundary Conditions (NSCBCs) are applied.

The coldflow LES strategy is validated for Conf. A against the experimental results by both Daviller et al. [15] and Kaiser et al. [16].

For the reactive LES, the flow downstream of the swirler is confined by a cylindrical tube of diameter $d=60 \mathrm{~mm}$. The BFER model [17] is applied for the reaction chemistry. It takes into account two reactions and six species. The computational mesh is refined in the region of the flame to $220 \mu \mathrm{m}$ based on a mesh convergence study. Dynamic flame thickening [13] is used so that the flame front spans over eight computational nodes. This corresponds to a thickening factor of approximately 3 for the operating point under investigation. The Boundary Conditions (BCs) for the temperature was set as follows: In the upstream plenum, the interior of the swirler and at the rod, the boundaries are iso-thermal $(T=292.0 \mathrm{~K})$, while downstream of the swirler the combustor walls temperature is adapted by a heat-loss BC. There, the parameters are set to account for a steel confinement of $10 \mathrm{~mm}$ thickness with an outside temperature of $500 \mathrm{~K}$.

GTP-19-1433 Thomas Ludwig Kaiser

\subsection{LSA}

Hydrodynamic LSA is based on a decomposition of velocity and pressure in at least two parts: One corresponding to the temporal mean and the other one to a coherent fluctuation. When additionally a part for stochastic fluctuations is considered, their interaction with the coherent structures can be taken into account. The decomposition is then called triple decomposition and reads

$$
\mathbf{u}=\overline{\mathbf{u}}+\widetilde{\mathbf{u}}+\mathbf{u}^{\prime}, p=\bar{p}+\widetilde{p}+p^{\prime}
$$

where the bar stands for the temporal mean, the tilde for a coherent fluctuation and the prime for the stochastic part. Inserting Eqn. 1 in the incompressible Navier-Stokes momentum equation and the continuity equation, yields the governing equations of LSA, which read

$$
\begin{aligned}
\frac{\partial \widetilde{\mathbf{u}}}{\partial t}+ & (\widetilde{\mathbf{u}} \cdot \nabla) \overline{\mathbf{u}}+(\overline{\mathbf{u}} \cdot \nabla) \widetilde{\mathbf{u}}+\langle(\widetilde{\mathbf{u}} \cdot \nabla) \widetilde{\mathbf{u}}\rangle-\overline{(\widetilde{\mathbf{u}} \cdot \nabla) \widetilde{\mathbf{u}}} \\
& +\left\langle\left(\mathbf{u}^{\prime} \cdot \nabla\right) \mathbf{u}^{\prime}\right\rangle-\overline{\left(\mathbf{u}^{\prime} \cdot \nabla\right) \mathbf{u}^{\prime}}=-\nabla \widetilde{p}+\frac{1}{\operatorname{Re}} \Delta \widetilde{\mathbf{u}}
\end{aligned}
$$

and

$$
\nabla \cdot \widetilde{\mathbf{u}}=0
$$

Here, $t$ is the time, the angle brackets denote a phase average and $\mathrm{Re}$ is the Reynolds number defined as

$$
\operatorname{Re}=\frac{U D}{v}
$$

The velocity $U$ and the distance $D$ are arbitrary velocity and length scales, which here are fixed to $U=50 \mathrm{~ms}^{-1}$ and $4.2 \mathrm{~mm}$. The fourth and fifth term on the Left Hand Side (LHS) in Eqn. (2) are product terms of the coherent fluctuations that arise from the nonlinear terms in the Navier-Stokes momentum equations. In LSA these are in general neglected, since we conclude that the flow perturbations remain small with respect to the underlying mean flow.

Furthermore, in Eqn. 2, non-vanishing product terms of stochastic fluctuations appear. They can be interpreted as the fluctuation in Reynolds stresses during the period of the coherent fluctuation [18]. This term is often modeled by an additional eddy viscosity $[19,20]$. Since both the temporal means and the fluctuations are known from LES, the Boussinesq approximation can be applied to estimate the eddy viscosity. Since this approach yields an eddy viscosity for every independent entry in the Reynolds stress tensor, the least mean squares of all eddy 
viscosities is applied, which is obtained by solving [21]

$$
v_{\mathrm{e}}=\frac{\left(-\overline{u_{i}^{\prime} u_{j}^{\prime}}+\frac{2}{3} K \delta_{i j}\right) \cdot\left(\frac{\partial \bar{u}_{j}}{\partial x_{i}}+\frac{\partial \bar{u}_{i}}{\partial x_{j}}\right)}{\left(\frac{\partial \bar{u}_{k}}{\partial x_{l}}+\frac{\partial \bar{u}_{l}}{\partial x_{k}}\right) \cdot\left(\frac{\partial \bar{u}_{k}}{\partial x_{l}}+\frac{\partial \bar{u}_{l}}{\partial x_{k}}\right)}
$$

where $i, j, k$ and $l$ are treated according to the Einstein notation and $K$ is the turbulent kinetic energy. LESs are based on a spatial filtering of the velocity fluctuations, i.e. eddies of large wave numbers are not resolved by this approach. This results in a slight decrease in turbulent kinetic energy in comparison with Direct Numerical Simulation (DNS) or experiments. Therefore, the eddy viscosity based on the equation above would be estimated slightly lower in LES than in DNS or experiments. We assume that the LES sub-grid scale viscosity, $v_{\mathrm{t}}$, accounts for this difference. Taking into account the molecular viscosity, $v_{\mathrm{m}}$, yields the total viscosity acting on the coherent structures:

$$
v=v_{\mathrm{e}}+v_{\mathrm{t}}+v_{\mathrm{m}}
$$

Taking advantage of the rotation symmetry, a BiGlobal modal approach can be formulated:

$$
[\widetilde{\mathbf{u}}, \widetilde{p}]=[\widehat{\mathbf{u}}, \widehat{p}] \exp (i m \theta-i \omega t)
$$

Here, $m$ is the azimuthal wave number, and $\omega$ is the complex circular frequency of the coherent structure. When inserting Eqn. (7) in the set of governing equations (Eqn. (2-3)), it becomes a Generalized Eigenvalue Problem (GEVP) where the eigenvalue is the circular frequency of an oscillations, $\omega$, and the pre-exponential factor in Eqn. (7) is the eigenvector:

$$
A[\widehat{\mathbf{u}}, \widehat{p}]=\omega B[\widehat{\mathbf{u}}, \widehat{p}]
$$

Spatial discretization of the matrices in the GEVP is performed by FreeFEM++ [22]. The computational mesh used for the LSA is illustrated in Fig. 5 exemplary for Conf. A. The mesh size in the coarse region is $0.9 \mathrm{~mm}$, while in the refined region it is $0.2 \mathrm{~mm}$. The BCs applied to the boundaries are shown in Tab 1 . The GEVP is the solved by the ARPACK package [23].

\section{Results}

\subsection{Experimental Results}

For Conf. A at a flow rate of $\dot{V}=3.41 \mathrm{~s}^{-1}$ an audible, loud whistling sound occurs in the experiment. A Fast Fourier Transform (FFT) of the microphone measurements show several distinct peaks in the pressure spectrum (see solid black line (-)

GTP-19-1433 Thomas Ludwig Kaiser in Fig. 6). A minor peak is visible at $f \approx 700 \mathrm{~Hz}$. The dominant spike appears at $f \approx 3150 \mathrm{~Hz}$. The remaining peaks are higher harmonics of the latter. Only the first higher harmonic is visible in Fig. 6.

In Conf. B, the dominant peak in the Sound Pressure Level (SPL) spectrum and its higher harmonics have disappeared (see dashed line (---) in Fig. 6). As a consequence, the wistling noise is no longer audible in the experiment. However, in place of the distinct dominant peak, a broadband elevation is visible in the SPL, which is located around $f \approx 2500 \mathrm{~Hz}$.

For both Conf. A and Conf. B, the cause of the peaks and the elevation could not be determined by the experiment. Proper Orthogonal Decomposition (POD), for example, based on the experimental PIV snapshots did not reveal any coherent structures. However, the experimental results yield a validation basis for the LES calculations, regarding both the time-mean quantities in the free stream fields and the peak frequencies occurring in the respective SPL spectrum.

\subsection{LES Time-Averaged Flows}

The temporal means of the cold flow LES are illustrated on the LHS of Fig 7 for Conf. A and on its Right Hand Side (RHS) for Conf. B. In Conf. A, the highest axial and tangential velocities occur within the primary injector, and they decay rapidly as the fluid reaches the dump plane. Outside of the injector, the highest velocity component is the radial one. As shown by the Line Integral Convolution (LIC) plot shown in the bottom row of Fig. 7, the flow directly attaches to the dump plane and no free stream jet evolves (see also Kaiser et al. [16]). On the jet center axis a large Central Recirculation Zone (CRZ) appears downstream of the primary injector. At the axis of the primary injector a large region of comparably low flow velocities exists.

When comparing the mean flows of Conf. B (see RHS of Fig. 7) with Conf. A, it appears that the rod leaves the flow field within the primary injector nearly unaffected. This might be because it covers mainly regions of very low flow velocities. Also here no free jet evolves, as the flow attaches to the dump plane after exiting the injector.

Finally, the influence of the geometry change on the flame shape is investigated based on reactive LES. The LHS of Fig. 8 shows the time-average of heat release rate for Conf. A. The flame is anchored in the central recirculation bubble inside the primary injector. Also in the reactive simulations the strong swirl causes the flow to remain attached on the dump plane, which leads to a very flat flame. The lateral confinement, which was added in the reactive simulation bends the flame in axial direction. In Conf. B (see RHS of Fig. 8) the flame shape resembles the one of Conf. A. This is in line with the observation, that mounting the central rod in the interior of the injector leaves the flow field nearly unchanged in the cold flow simulations. Only the region, which shows the highest average reaction rate 
in Conf. A is covered by the central rod in Conf. B.

\subsection{LES Cold Flow Dynamics}

In order to investigate the dynamics of the cold flow, a FFT of the pressure probe measurements conducted in the primary injector as well as Dynamic Mode Decompositions (DMDs) of three dimensional LES snapshots are performed. Figure 9 shows the pressure spectra at the probe in the interior of the primary injector ${ }^{1}$. The spectra agree well with the experimental observations. For Conf. A ( - ) a dominant peak occurs at $f \approx 3100 \mathrm{~Hz}$, which is accompanied by several higher harmonics. As in the experiment, the dominant peak disappears in Conf. B and is replaced by a broadband elevation of the pressure spectrum, which also in the numerical results is located around $f \approx 2500 \mathrm{~Hz}$.

The same behavior is also found for the DMD pressure amplitude spectrum, which is illustrated in Fig. 10 (See e.g. Tu et al. [24] for the calculation of a DMD amplitude spectrum). In the DMD amplitude spectrum a dominant peak is found at $f \approx 3030 \mathrm{~Hz}$ for Conf. A, while in Conf. B again a broadband elevation at slightly lower frequencies can be observed. The peak of this broadband elevation is at $f \approx 2680 \mathrm{~Hz}$. Even the small peak at $700 \mathrm{~Hz}$ seen in the experimental SPL is seen in the DMD amplitude spectrum. Due to the unambiguous agreement between the frequencies occurring in the experimental SPL spectrum and the pressure spectrum based both on FFT of a probe data and the three-dimensional LES snapshots, we assume that the dynamics of the flow are correctly reproduced by the LES.

The origin of the perturbations could not be explained by experimental means, however, since the dynamics of the flow appear to be reproduced by the LES we can analyze the DMD modes corresponding to the peaks in the DMD amplitude spectrum. The minor peak at $f \approx 700 \mathrm{~Hz}$ can be related to an axisymetric instability and is not further investigated here. The mode corresponding to the dominant peak in the pressure spectrum however is related to a single helical instability, i.e. a PVC. Its mode shape is illustrated on the LHS of Fig. 11. The fluctuations appear mainly in the interior of the primary injector, which is why they remain not accessible by experimental PIV.

Also the elevation in the DMD amplitude spectrum of Conf. B is caused by a single helical structure. The mode shapes at the peak of the spectrum ((---) in Fig. 10) are illustrated on the RHS of Fig. 11. It appears that the mode shapes in Conf. B agree well with the mode shapes of Conf. A, but are somewhat more erratic than in the case without the central rod.

\footnotetext{
${ }^{1}$ Note that the microphone location in the experiment is outside of the compu-
tional domain. A direct comparison with the experimental sound pressure level

${ }^{1}$ Note that the microphone location in the experiment is outside of the compu-
tational domain. A direct comparison with the experimental sound pressure level therefore is not possible
} GTP-19-1433 Thomas Ludwig Kaiser

where $S$ corresponds to the PSD, $\omega_{0}$ to the natural circular frequency of the mode, $v$ is the dampening rate of the mode and $\frac{\Gamma}{2 \pi}$ is the intensity of turbulent forcing. These three variables can be cle $(-)$ are related to a negative temporal growth rate $\left(\omega_{i}^{*}<0\right)$. One mode, however, stands out with a complex frequency of $\omega=1.89+0.01 i$ and is marked by a star $(---)$. This corresponds to a marginally positive growth rate (and therefore an instability at its limit cycle) and a real frequency which overestimates the frequency seen in the LES by approximately $18 \%$. The mode shapes of this BiGlobal LSA mode are illustrated on the LHS of Fig. 13. Outside the primary injector, the LSA mode shapes agree well with the DMD modes extracted from LES. Within the primary injector, this holds for the axial velocity component and the pressure, while discrepancies occur for the radial and azimuthal velocity components. Fig. 14 illustrates the region of highest internal feedback or structural sensitivity, $\lambda$. It shows that the origin of the instability is at the exit plane of the primary injector for Conf. A.

The spectrum for Conf. B is illustrated in Fig. 15 by the round marks. In contrast to Conf. A, all the eigenmodes are related to a negative temporal growth rate. Nevertheless, the eigenvalue at $\omega \approx 1.4-0.2 i(-)$ can be related to the DMD mode shapes seen for Conf. B and therefore also to the BiGlobal mode in Conf. A. The arrow marks the shift of the eigenvalue due to the geometry change from Conf. A (-- ) to Conf. B (-). The shapes of the respective mode are illustrated on the RHS of Fig. 13. A comparison with the DMD mode (see Fig. 11) confirms that the BiGlobal mode causes the elevation in the experimental SPL, the LES-pressure and the LES-DMD amplitude spectra.

Despite its negative BiGlobal temporal growth rate, the mode occurs in the experiment and the LES. We will now test the assumption, that this linearly stable mode is excited by intermittent turbulent forcing. Noiray and Schuermanns [25] investigate this effect and show that it can lead to a broadband increase in the spectral plots, as observed in our work. They derive an approximate for the increase in the Power Spectral Density (PSD), which holds for modes of negative temporal growth and if turbulent forcing is weak enough to neglect non-linear forcing effects. This relation is given by

$$
S=\frac{1}{2 \pi} \frac{\Gamma}{\left(\omega_{0}^{2}-\omega_{\mathrm{PSD}}^{2}\right)^{2}+4 v^{2} \omega_{\mathrm{PSD}}^{2}}
$$


estimated by fitting $S$ to the elevation in the PSD spectrum caused by a linearly stable mode in case of background turbulence. Figure. 16 compares the PSD of the pressure measured in the interior of the primary injector ${ }^{2}$ with a corresponding fit of Eqn. (9). The model parameters leading to the fit are $\omega_{0}=1.37, v=0.15$ and $\Gamma=7500$. Both the natural frequency and the dampening rate, which correspond respectively to the real and imaginary part of an eigenvalue in the LSA correspond very well with the results obtained by LSA. The fitting parameters therefore confirm the assumption that the linearly stable mode is excited by intermittent forcing and at the same time validate the results of LSA obtained for Conf. B.

A comparison of the structural sensitivity maps of Conf. A (LHS in Fig. 14) and Conf. B (RHS in Fig. 14) yields a possible explanation for the growth rate reducing impact of the rod: Not only the mean flow field remains nearly unaffected by the presence of the rod, also the location of highest internal feedback is only marginally affected. Yet, the rod covers large parts of the area where in Conf. A the internal feedback is significant. Therefore, the rod apparently prevents the feedback of the PVC and therefore leads to its suppression.

The agreement between the mode shapes based on DMD and LSA is unambiguously better in Conf. B in comparison with Conf. A, especially for the radial and the azimuthal velocity component. Also the frequency estimated by the BiGlobal approach is very close to the frequency seen in LES for Conf. B with an error of only $1 \%$ and hence is by far more precise than in Conf. A.

\section{CONCLUSION}

The flow in a two-staged, counter rotative, industrial fuel injection system is investigated by means of experiments, LES and LSA. Flows in two configurations of different geometry are compared with respect to their hydrodynamic instability. In the first configuration (Conf. A), a loud whistling sound occurs in the experiment and the SPL spectrum based on microphone measurements shows a dominant spike. The cause, however, could not be clarified by experimental means. DMD modes of LES snapshots show that the origin of the whistling in the experiment is a PVC in the interior of the primary injector. BiGlobal LSA finds a marginally unstable mode, which corresponds to an instability at its limit cycle. The origin of the mode, also often referred to as wave maker, is found to be at the exit plane of the primary injector, which is in line with local stability analysis (see Kaiser et al. [16]). The frequency of the mode is predicted with reasonable accuracy and the mode shapes, while agreeing very well for all components outside of the primary injector, slightly differ for the

\footnotetext{
${ }^{2}$ Note that the time series of the pressure measurements is too short for a fully converged PSD. In order to obtain a suitable target for the fitting, a sliding average (filter order of 3) was applied for the numerical PSD illustrated in Fig. 16
}

GTP-19-1433 Thomas Ludwig Kaiser radial and azimuthal velocity component from the DMD modes in the interior of the primary injector.

In the second configuration (Conf. B), a rod is mounted as a center body in the interior of the primary injector. It is demonstrated by non-reactive LES that the mean flow is only marginally modified by this large geometry change. By using reactive LESs, the impact of the central rod on the mean flame shape is furthermore analyzed. By comparing the resulting time-averaged flame shapes, it is found that the shapes remains mainly unaffected by the presence of the rod.

While the mean properties of the flame are marginally affected, the cold flow experiment on the other hand shows that the rod has a significant impact on the dynamics of the system, since the whistling noise observed in Conf. A disappears for Conf. B. In the SPL spectrum, the spike is replaced by a broadband elevation of the spectrum at a slightly lower frequency. The LES reproduces this behavior in both the pressure spectrum and the DMD amplitude spectrum. LSA shows that the mode which occurs in Conf. A is of much lower growth rate and frequency when the rod is applied. As a consequence, the mode is now linearly stable. The frequency of this stable mode is very close to the measured peak frequency. Furthermore, the dampening rate of the mode was extracted from the SPL spectrum, which is in good agreement with the value obtained by LSA. Also for Conf. $\mathrm{B}$, the mode shapes based on DMD and LSA are in very good agreement. An analysis of the structural sensitivity suggests that the rod interferes with the feedback mechanism of the PVC leading to its suppression.

The study demonstrates that mounting a center body in the interior of a swirled injector can stabilize the PVC without having necessarily a significant impact on the flame shape. We thereby show that geometry changes in general, which do not necessarily have a large impact on the mean flow, can have a significant effect on the formation process of dynamic flow structures. In this context, the study underlines the value of the LSA method for the combustion community, since it correctly predicts the growth rates, frequencies and mode shapes in both configurations. A subsequent, important topic in this context is geometry optimization by the adjoint method as proposed by Tammisola and Juniper [10]. In the present configuration for example, this method could be applied to adapt the geometry of the center body in such a way that either the PVC is suppressed with an even more marginal impact on the mean flow, or that even in the presence of a center body a PVC is generated.

\section{ACKNOWLEDGMENT}

This work was granted access to the high-performance computing resources of CINES under the allocation x20152b7036 made by Grand Equipement National de Calcul Intensif. The research leading to these results has received funding from the European Research Council under the European Unions Sev- 
enth Framework Program (FP/2007-2013)/ERC Grant agreement ERC-AdG 319067-INTECOCIS and the Deutsche Forschungsgemeinschaft (DFG) under project number OB 402/4-3. The authors also would like to thank Moritz Sieber for sharing his expertise on stochastic excitation of linearly stable modes.

\section{REFERENCES}

[1] Stöhr, M., Boxx, I., Carter, C. D., and Meier, W., 2012. "Experimental study of vortex-flame interaction in a gas turbine model combustor". Combustion and flame, 159(8), pp. 2636-2649.

[2] Poinsot, T. J., Trouve, A. C., Veynante, D. P., Candel, S. M., and Esposito, E. J., 1987. "Vortex-driven acoustically coupled combustion instabilities". Journal of Fluid Mechanics, 177, pp. 265-292.

[3] Candel, S. M., 1992. "Combustion instabilities coupled by pressure waves and their active control". Symposium (International) on Combustion, 24(1), pp. 1277 - 1296. TwentyFourth Symposium on Combustion.

[4] Qadri, U., Mistry, D., and Juniper, M., 2011. "Sensitivity Analysis of Spiral Vortex Breakdown". In Colloquium 525 - Instabilities and transition in three-dimensional flows with rotation, EU-ROMECH, pp. hal-00600349.

[5] Giannetti, F., and Luchini, P., 2007. "Structural sensitivity of the first instability of the cylinder wake". Journal of Fluid Mechanics, 581, pp. 167-197.

[6] Kuhn, P., Moeck, J. P., Paschereit, C. O., and Oberleithner, K., 2016. "Control of the precessing vortex core by open and closed-loop forcing in the jet core". In ASME Turbo Expo 2016: Turbomachinery Technical Conference and Exposition, American Society of Mechanical Engineers, pp. V04BT04A036-V04BT04A036.

[7] Lückoff, F., Sieber, M., and Oberleithner, K., 2018. “Openloop control of the precessing vortex core in a swirlstabilized combustor: Impact on flame shape and flame stability". In ASME Turbo Expo 2018: Turbomachinery Technical Conference and Exposition, American Society of Mechanical Engineers, pp. V04AT04A029-V04AT04A029.

[8] Chomaz, J.-M., 2005. "GLOBAL INSTABILITIES IN SPATIALLY DEVELOPING FLOWS: Non-Normality and Nonlinearity". Annual Review of Fluid Mechanics, 37, pp. 357-392.

[9] Strykowski 'a N, P. J., and Sreenivasan ', D. K. R., 1990. "On the formation and suppression of vortex 'shedding ' at low Reynolds numbers". Journal of Fluid Mechanics, 218, pp. 71-107.

[10] Tammisola, O., and Juniper, M. P., 2015. "Adjoint sensitivity analysis of hydrodynamic stability in a gas turbine fuel injector". In ASME Turbo Expo 2015: Turbine Technical Conference and Exposition, American Society of Mechanical Engineers, pp. V04AT04A057-V04AT04A057.
[11] Paschereit, C. O., Flohr, P., Knpfel, H., Geng, W., Steinbach, C., Stuber, P., Bengtsson, K., and Gutmark, E., 2002. "Combustion control by extended ev burner fuel lance". In ASME Turbo Expo 2002: Power for Land, Sea, and Air, American Society of Mechanical Engineers, pp. 721-730.

[12] Poinsot, T., 2005. The AVBP Handbook. CERFACS, Toulouse.

[13] Colin, O., Ducros, F., Veynante, D., and Poinsot, T., 2000. "A thickened flame model for large eddy simulations of turbulent premixed combustion". Physics of fluids, 12(7), pp. $1843-1863$.

[14] Nicoud, F., Toda, H. B., Cabrit, O., Bose, S., and Lee, J., 2011. "Using singular values to build a subgrid-scale model for large eddy simulations". Physics of Fluids, 23(8), p. 085106.

[15] Daviller, G., Brebion, M., Xavier, P., Staffelbach, G., Müller, J.-D., and Poinsot, T., 2017. "A mesh adaptation strategy to predict pressure losses in les of swirled flows". Flow, Turbulence and Combustion, 99(1), pp. 93-118.

[16] Kaiser, T. L., Poinsot, T., and Oberleithner, K., 2018. "Stability and Sensitivity Analysis of Hydrodynamic Instabilities in Industrial Swirled Injection Systems". Journal of Engineering for Gas Turbines and Power, 140(5), p. 51506.

[17] Franzelli, B., Riber, E., Gicquel, L. Y., and Poinsot, T., 2012. "Large eddy simulation of combustion instabilities in a lean partially premixed swirled flame". Combustion and flame, 159(2), pp. 621-637.

[18] Hussain, A. K. M. F., and Reynolds, W. C., 1970. "The mechanics of an organized wave in turbulent shear flow". Journal of Fluid Mechanics, 41(2), pp. 241-258.

[19] Tammisola, O., and Juniper, M. P., 2016. "Coherent structures in a swirl injector at $\mathrm{Re}=4800$ by nonlinear simulations and linear global modes". Journal of Fluid Mechanics, 792, pp. 620-657.

[20] Rukes, L., Paschereit, C. O., and Oberleithner, K., 2016. "An assessment of turbulence models for linear hydrodynamic stability analysis of strongly swirling jets". European Journal of Mechanics, B/Fluids, 59, pp. 205-218.

[21] Ivanova, E. M., Noll, B. E., and Aigner, M., 2013. "A numerical study on the turbulent Schmidt numbers in a jet in crossflow". Journal of Engineering for Gas Turbines and Power, 135(1), pp. 011505/1-011505/10.

[22] Hecht, F., 2012. "New development in FreeFem++". Journal of Numerical Mathematics, 20(3-4), pp. 251-265.

[23] Lehoucq, R., Sorensen, D., and Yang, C., 1997. Arpack users' guide: Solution of large scale eigenvalue problems with implicitly restarted Arnoldi methods.

[24] Tu, J. H., Rowley, C. W., Luchtenburg, D. M., Brunton, S. L., and Kutz, J. N., 2013. "On dynamic mode decomposition: theory and applications". arXiv preprint arXiv:1312.0041.

[25] Noiray, N., and Schuermans, B., 2013. "Deterministic 
TABLE 1: Boundary conditions for the perturbations; $\mathbf{n}$ is the normal vector of the boundary.

\begin{tabular}{|l|c|c|c|c|}
\hline & Axis & Walls & Inlet & Outlet \\
\hline radial vel. & $\widetilde{u}_{r}=0$ & $\widetilde{u}_{r}=0$ & $\frac{\partial \widetilde{u}_{r}}{\partial \mathbf{n}}=0$ & $\widetilde{u}_{r}=0$ \\
\hline azimuthal vel. & $\widetilde{u}_{t}=0$ & $\widetilde{u}_{r}=0$ & $\frac{\partial \widetilde{u}_{t}}{\partial \mathbf{n}}=0$ & $\widetilde{u}_{t}=0$ \\
\hline axial vel. & $\frac{\partial \widetilde{u}_{z}}{\partial \mathbf{n}}=0$ & $\widetilde{u}_{r}=0$ & $\frac{\partial \widetilde{u}_{z}}{\partial \mathbf{n}}=0$ & $\widetilde{u}_{z}=0$ \\
\hline pressure & $\frac{\partial \widetilde{p}}{\partial \mathbf{n}}=0$ & $\frac{\partial \widetilde{p}}{\partial \mathbf{n}}=0$ & $\frac{\partial \widetilde{p}}{\partial \mathbf{n}}=0$ & $\widetilde{p}=0$ \\
\hline
\end{tabular}

quantities characterizing noise driven hopf bifurcations in gas turbine combustors". International Journal of NonLinear Mechanics, 50, pp. 152-163.

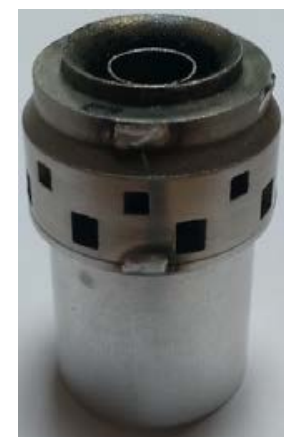

(a) Configuration $\mathrm{A}$

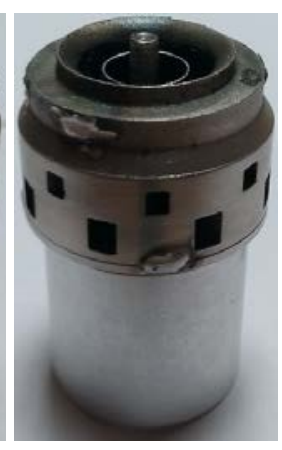

(b) Configuration B
FIGURE 1: Both configurations of the two-staged counterrotative injection system

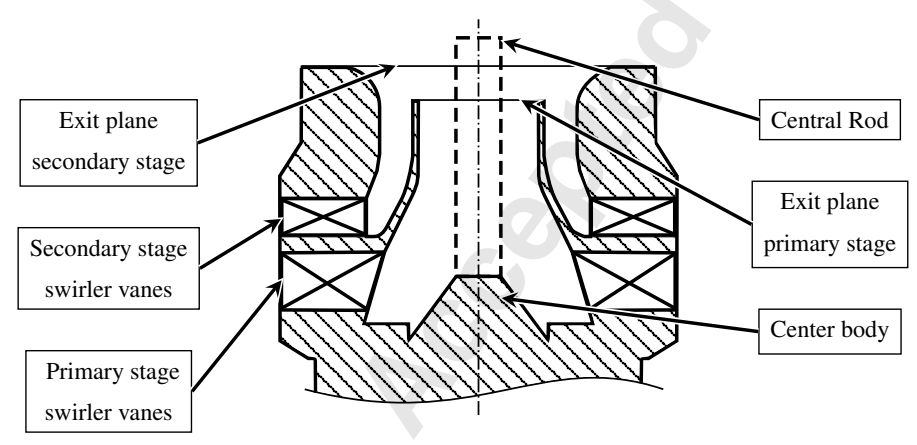

FIGURE 2: Sketch of the cross section of the swirler

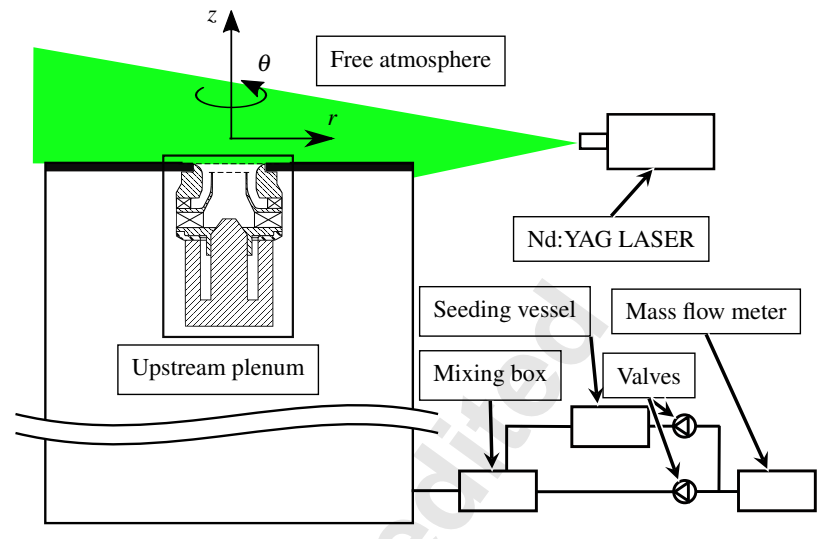

FIGURE 3: Schematic set up of the experiment

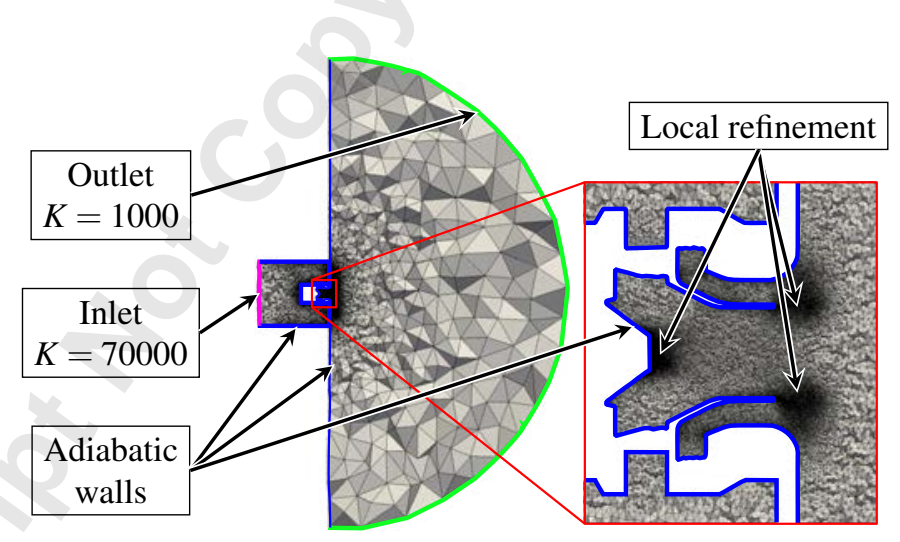

FIGURE 4: Numerical domain with the mesh used for calculations of Conf. A

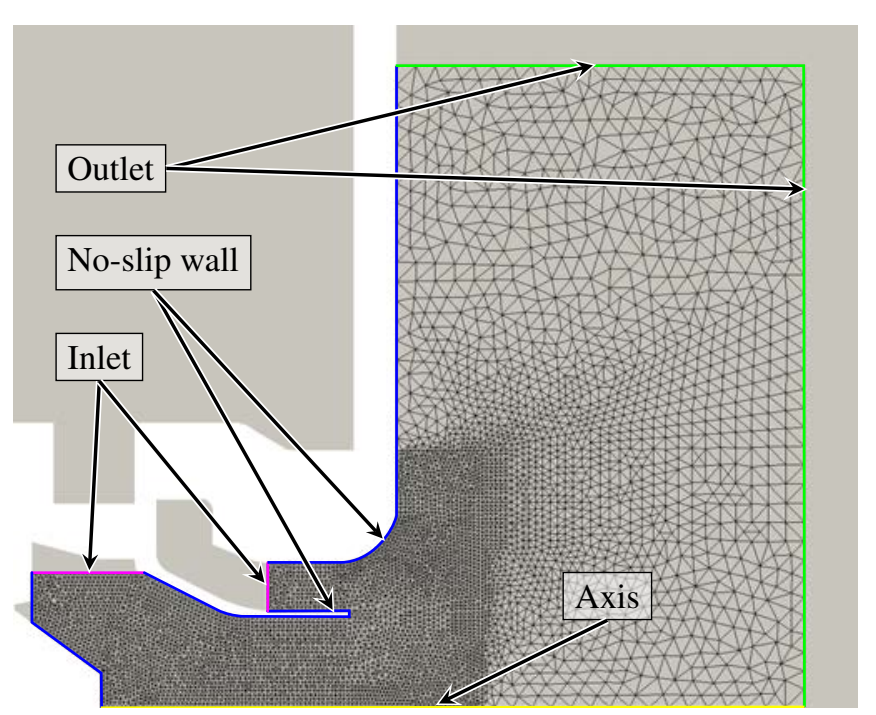

FIGURE 5: Numerical domain and grid for the BiGlobal LSA, exemplary for Conf. A 


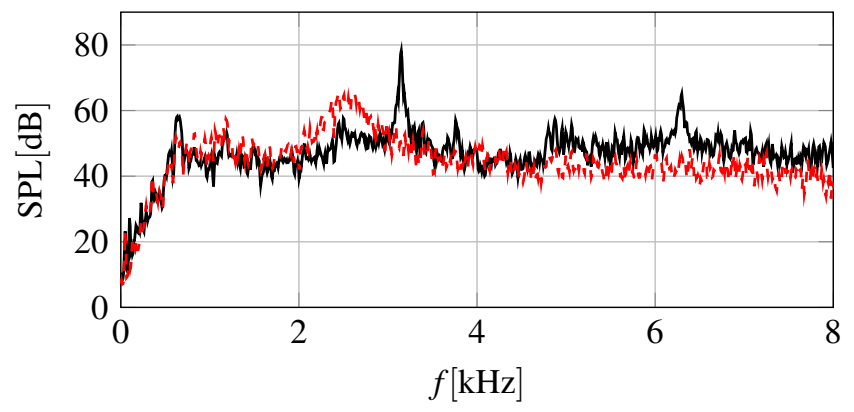

FIGURE 6: Experimental SPLs measured at $x=0$ and $r=$ $300 \mathrm{~mm}$ for Conf. A (-) and Conf. B (- - - )
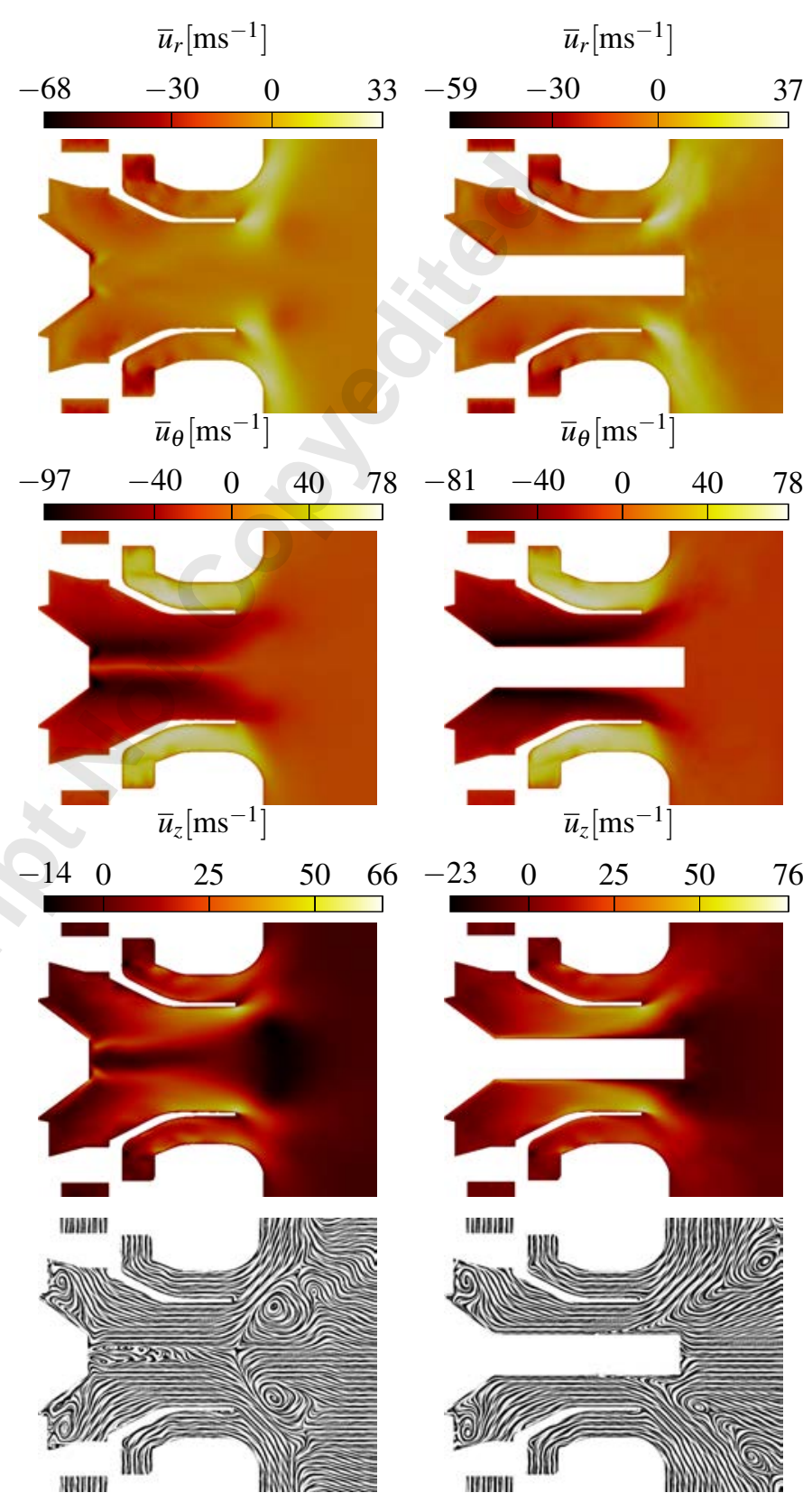

FIGURE 7: Temporal means of LES velocity components and LIC based on the radial and axial velocity component for Conf. A (LHS) and Conf. B (RHS) 


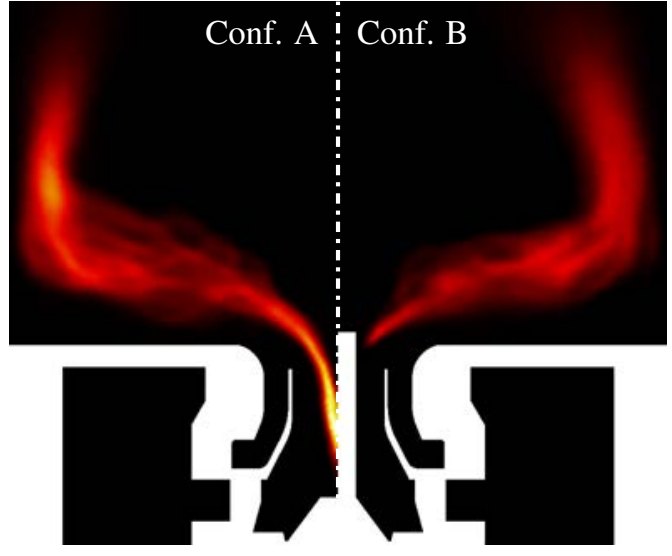

FIGURE 8: LES flame shapes indicated by the time average of the heat release for Conf. A and Conf. B; $\Phi=0.75, \dot{V}=3.41 \mathrm{~s}^{-1}$

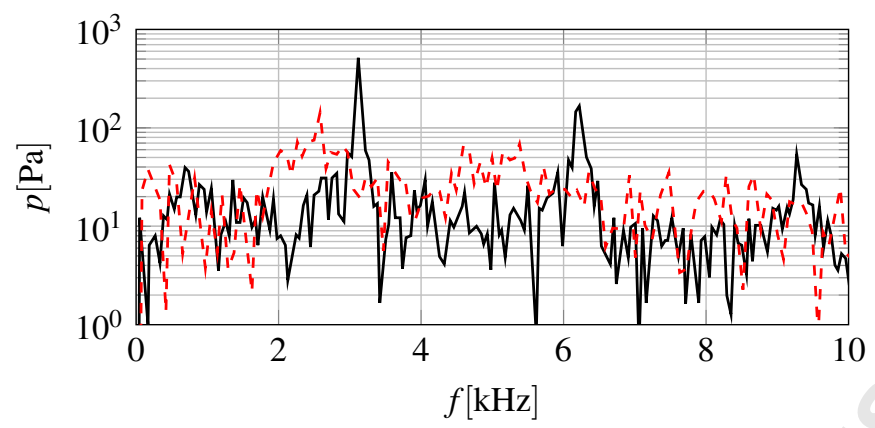

FIGURE 9: Fourier transform of numerical pressure measurements within the primary injector for Conf. A (-) and Conf. B (- - - )

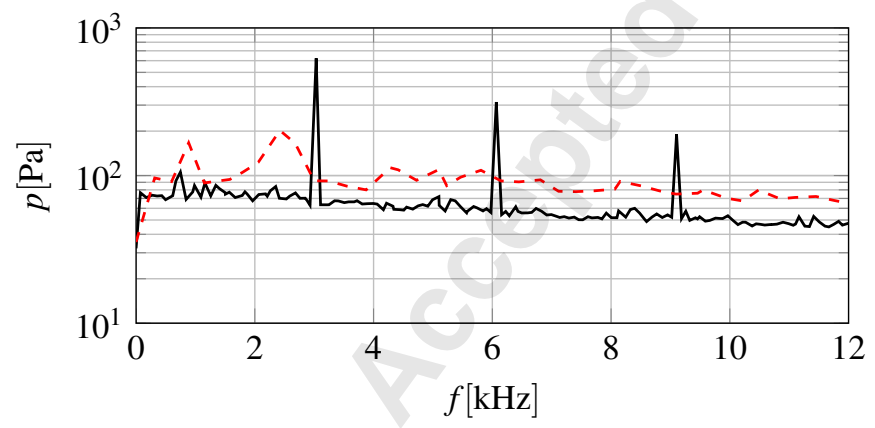

FIGURE 10: DMD amplitude spectra for Conf. A ( $*$ and Conf. B (•) based on three dimensional LES snapshots

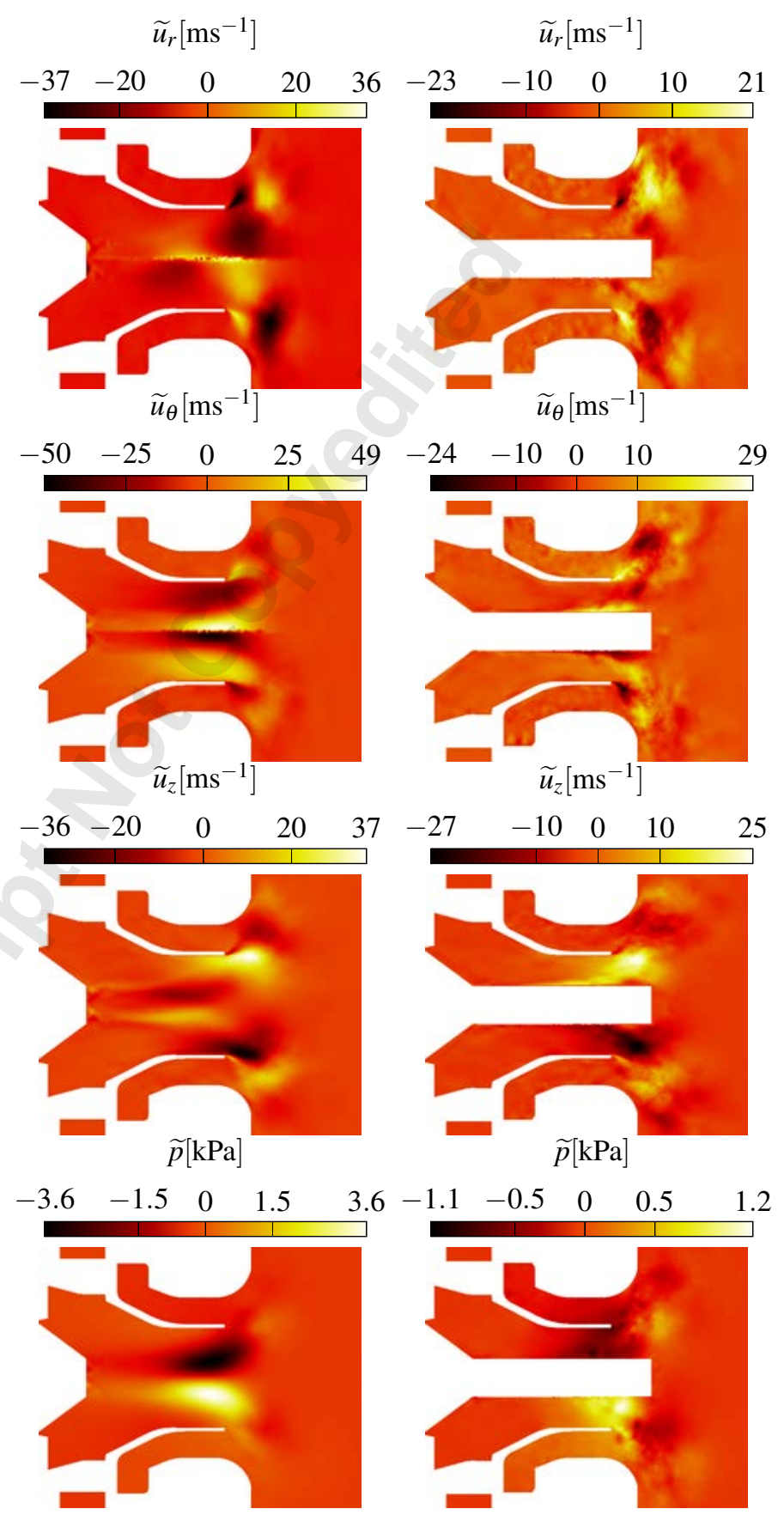

FIGURE 11: DMD mode shapes of the velocity components and pressure for Conf. A (LHS) and Conf. B (RHS); The mode illustrated for Conf. A corresponds to the dominant peak (---) and for Conf. B to the highest elevation $(*)$ in the corresponding DMD amplitude spectra illustrated in Fig. 10 


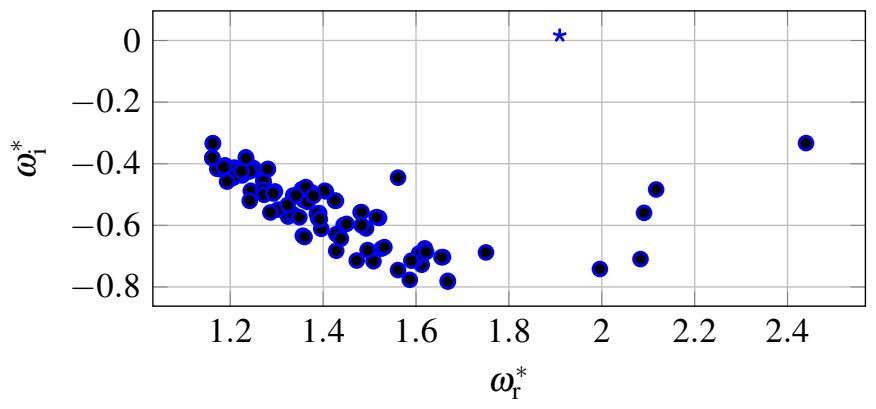

FIGURE 12: BiGlobal spectrum for Conf. A: Marginally unstable $(\bullet)$ and stable $(\bullet)$

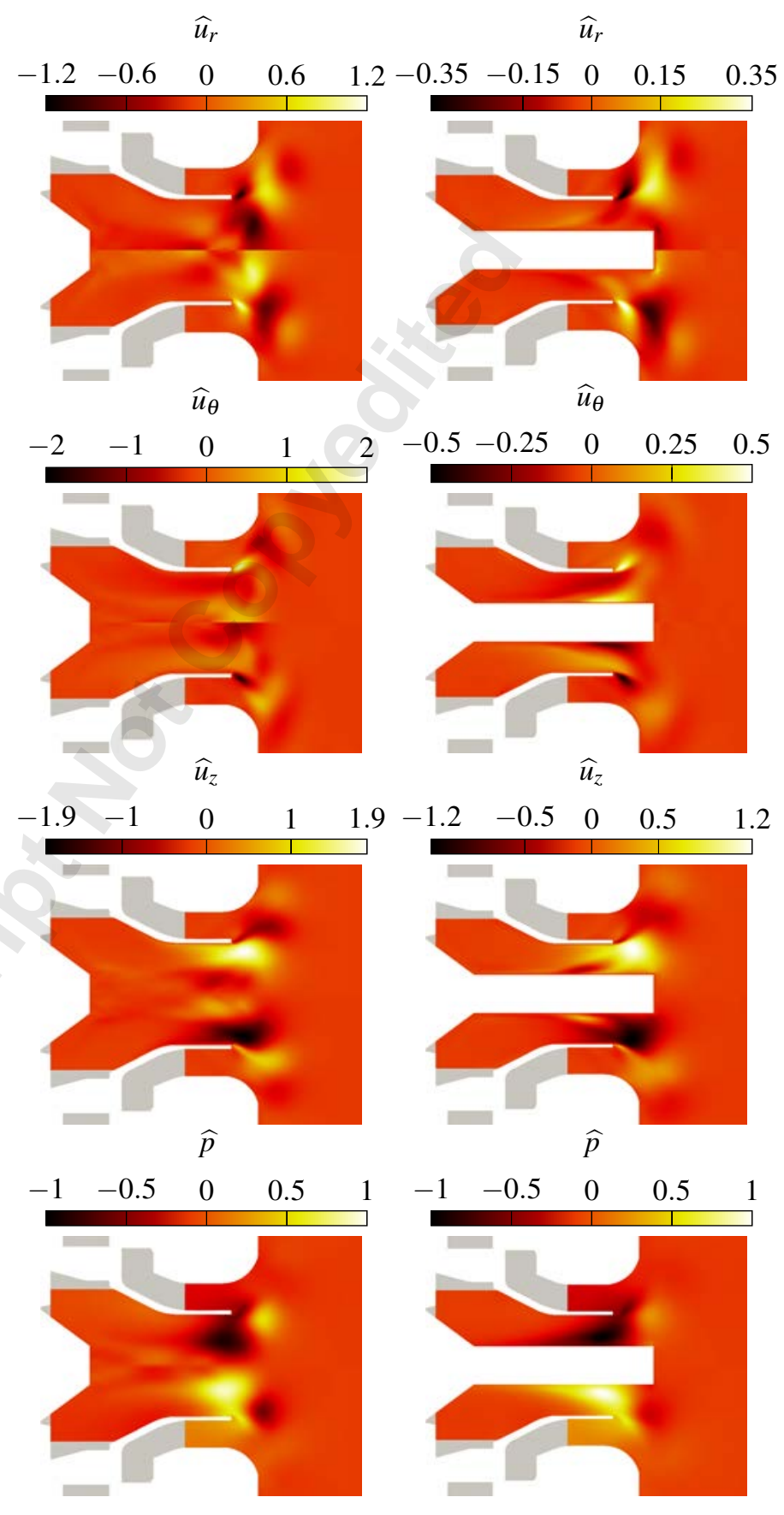

FIGURE 13: LSA mode shapes of the velocity components and pressure for Conf. A (LHS) and Conf. B (RHS); The mode illustrated for Conf. A corresponds to the marginally unstable mode ( $\bullet)$ in Fig. 12 and for Conf. B the mode indicated by (o) in Fig. 15 

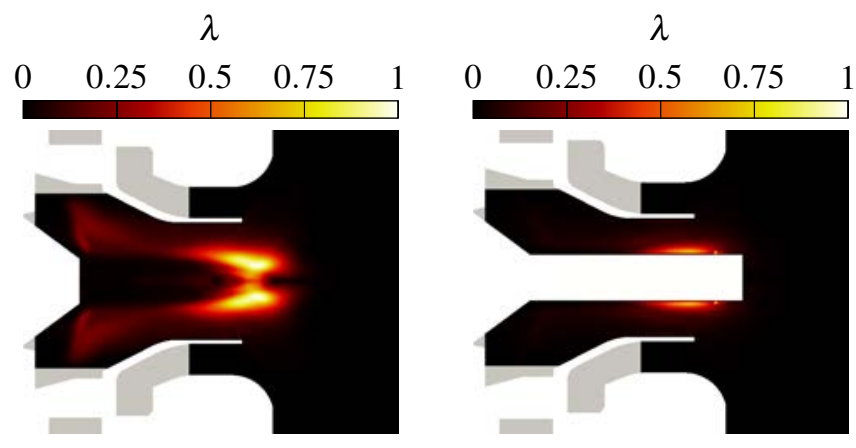

FIGURE 14: Structural sensitivity map for the BiGlobal modes in Conf. A (LHS) and Conf. B (RHS)

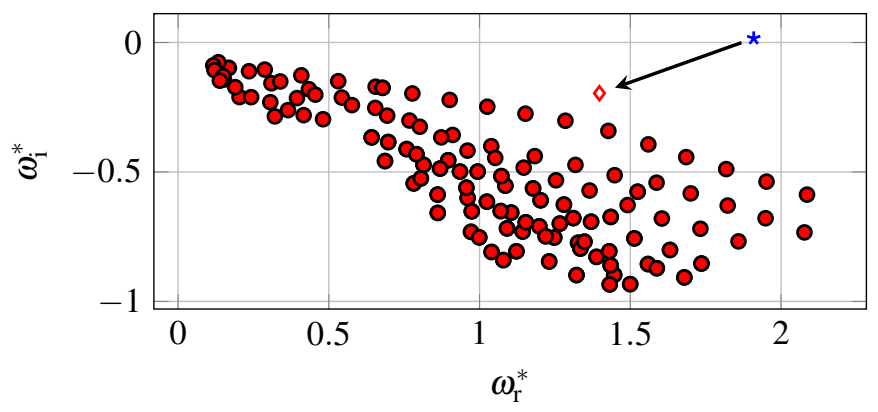

FIGURE 15: BiGlobal spectrum for Conf. B against unstable mode from Conf. A ( () ; The in Conf. A unstable mode is shifted by the presence of the rod (*); the remaining stable modes are marked by 0

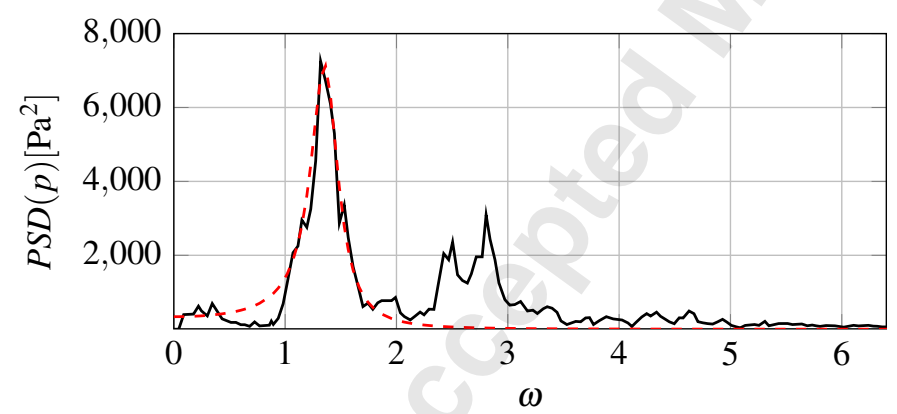

FIGURE 16: PSD of numerical pressure in the interor of the primary injector (-) and fitted curve (- - ) based on Eqn. (9); Fitting parameters: $\omega_{0}=1.37, v=0.15, \Gamma=7500$ 